\title{
The Rice Floral Repressor Early flowering1 Affects Spikelet Fertility By Modulating Gibberellin Signaling
}

\author{
Choon-Tak Kwon ${ }^{1}$, Suk-Hwan Kim¹, Dami Kim ${ }^{1}$ and Nam-Chon Paek ${ }^{1,2^{*}}$
}

\begin{abstract}
Background: Gibberellic acid (GA; or gibberellin) affects the development of floral organs, especially anthers and pollen, and perturbation of development of male floral organs can cause sterility. Many studies of GA signaling have concentrated on anther development, but the effect of GA on grain production remains to be examined.

Results: Using a cross of 'Milyang23 (M23)', which has a functional allele of Early flowering1 (EL1), and 'H143', which has a nonfunctional el1 allele, we generated heterogeneous inbred family-near isogenic lines (HNILs) that are homozygous for EL1 [HNIL(M23)] or el1 [HNIL(H143)]. Here, we found that HNIL(H143) exhibited anther deformities and low pollen viability. The expression of GAMYB, a major activator of GA signaling, and its downstream genes CYP703A3 and KAR, mainly involved in pollen formation, increased abnormally during spikelet development; this activation of GA signaling may cause the sterility. To confirm the negative effect of the el1 mutation on spikelet fertility, we examined a line carrying a T-DNA insertion el1 mutant [hereafter ZH11(e/1)] and its parental cultivar 'Zhonghua11 (ZH11)'. ZH11(e/1) showed nearly identical defects in anther development and pollen viability as HNIL(H143), leading to decreased seed setting rate. However, the elite japonica cultivar Koshihikari, which has a nonfunctional el1 allele for early flowering in long days, produces fertile spikelets and normal grain yields, like other elite japonica cultivars. This indicates that as-yet-unknown regulator(s) that can overcome the male sterile phenotype of the ell mutation must have been introduced into Koshihikari.

Conclusions: The el1 mutation contributes to early flowering in japonica rice under long days but fails to limit GA signaling, thus negatively affecting spikelet fertility, which results in a loss of grain yield. Thus, EL1 is essential for photoperiod sensitivity in flowering as well as spikelet fertility in grain production.
\end{abstract}

Keywords: Rice; Early flowering1; Spikelet fertility; Seed setting rate; Anther development; Pollen viability; GA signaling

\section{Background}

Rice (Oryza sativa L.) is a major staple food for more than a half of the world population, mainly in Asia. Enhancing the production of rice will require improvements of the major yield components, such as the number of panicles per plant, the number of spikelets per panicle, the seed setting rate, and the weight of each grain. Furthermore, these improvements must maintain the balance of yield-related traits; for example, inordinate tillering

\footnotetext{
* Correspondence: ncpaek@snu.ac.kr

'Department of Plant Science, Plant Genomics and Breeding Institute, and Research Institute of Agriculture and Life Sciences, Seoul National University, Seoul 151-921, Republic of Korea

${ }^{2}$ Crop Biotechnology Institute, GreenBio Science and Technology, Seoul National University, Pyeongchang 232-916, Republic of Korea
}

often decreases the grain yield per plant, because late-producing, infertile tillers compete for resources with the main, fertile shoot, thus decreasing spikelet fertility or seed setting rate (Liu et al. 2013).

Spikelet fertility, an important component of grain production, is influenced by environmental conditions and genetic background. Anther development and pollen viability particularly affect spikelet fertility (Liu et al. 2013), and numerous genetic studies have shown a significant relationship between the expression of the genes controlling the development of anthers and pollen and the fertility of the spikelet. For instance, normal anther formation and dehiscence in rice require ANTHER INDEHISCENCE1 (AID1) and the SUMO E3 ligase encoded by OsSIZ1 (Zhu et al. 2004; Thangasamy et al. 2011). 
Moreover, phytohormones and the genes involved in hormone biosynthesis or signal pathways affect anther dehiscence and pollen maturation. In Arabidopsis (Arabidopsis thaliana), auxin and jasmonic acid have crucial functions in fertility, acting by regulating anther and pollen development (Park et al. 2002; Cecchetti et al. 2008). Gibberellic acid (GA; also gibberellin) also has important roles in germination and floral organ development, acting by regulating stamen and anther formation (Nester and Zeevaart 1988; Ritchie and Gilroy 1998; Goto and Pharis 1999; Woodger et al. 2003). Moreover, GA is critical for pollen formation and pollen tube growth (Singh et al. 2002; Chhun et al. 2007), and GA signaling pathways are conserved between rice and Arabidopsis (Hedden and Phillips 2000; Fleet and Sun 2005). In particular, the homology of several factors involved in GA signaling and the strong similarities in stamen development between Arabidopsis and rice indicate the existence of conserved pathways of anther and pollen development within angiosperms (Chen et al. 2005; Itoh et al. 2005b; Wilson and Zhang 2009; Plackett et al. 2011).

Phenotypic screens, such as those for GA-insensitive dwarf or slender-type mutants in Arabidopsis and rice, have identified several genes necessary for GA-responsive signaling (Aya et al. 2009). The identification and functional analysis of the mutated genes has improved our understanding and allowed the construction of a model of GA signal transduction (Ueguchi-Tanaka et al. 2007b). In GA signal transduction in rice, the biologically active $\mathrm{GA}_{4}$ directly binds to the soluble GA receptor GA INSENSITIVE DWARF1 (GID1) (Ueguchi-Tanaka et al. 2005) and the $\mathrm{GA}_{4}$-GID1 complex physically interacts with the DELLA protein SLENDER RICE1 (SLR1) (UeguchiTanaka et al. 2007a), leading to degradation of SLR1 through the $\mathrm{SCF}^{\mathrm{GID} 2}$ complex in the $26 \mathrm{~S}$ proteasome pathway (Sasaki et al. 2003; Gomi et al. 2004). SLR1 controls GA signaling by negatively regulating the expression of GA-responsive genes such as GAMYB (Ikeda et al. 2001; Itoh et al. 2002; Aya et al. 2009). The central negative regulator of GA signaling, SLR1 exists in phosphorylated and non-phosphorylated forms in vivo (Sasaki et al. 2003; Gomi et al. 2004; Itoh et al. 2005a). Early work proposed that the degradation of SLR1 by the SCF ${ }^{\mathrm{GID} 2}$ complex depends on its phosphorylation (Sasaki et al. 2003; Gomi et al. 2004). However, subsequent work showed that non-phosphorylated SLR1 interacts with GID2, indicating that the phosphorylation of SLR1 is not essential for its degradation (Itoh et al. 2005a).

Several reports have described the post-translational modification of SLR1 protein, but few reports have examined its modifiers. Rice SPINDLY, encoding an O-linked $\mathrm{N}$-acetylglucosamine transferase, does not control the stability of SLR1 but probably participates in the repression of SLR1 activity in GA signaling
(Shimada et al. 2006). Early flowering1 (EL1), encoding casein kinase I (CKI), is associated with the negative regulation of GA signaling, acting by phosphorylating SLR1 protein and activating its function in repressing gene expression (Dai and Xue 2010). Although the phosphorylation of SLR1 by EL1/CKI contributes to maintaining SLR1 protein stability and activity (Dai and Xue 2010), the function of phosphorylated SLR1 protein still remains unclear.

The degradation of DELLA proteins caused by perception of GA triggers the transcription of downstream genes involved in GA signaling (Itoh et al. 2002; Murray et al. 2003; Ueguchi-Tanaka et al. 2007b). GAMYB, the main transcription factor that initiates GA signaling, acts as a positive transcriptional regulator of the $\alpha$-amylase gene in the cereal aleurone during germination (Gubler et al. 1995; Gubler et al. 1999; Gubler et al. 2002; Kaneko et al. 2004). A little over a decade ago, several reports showed that GAMYB is also essential for anther and pollen development in angiosperms (Murray et al. 2003; Achard et al. 2004; Kaneko et al. 2004; Tsuji et al. 2006; Chhun et al. 2007; Aya et al. 2009). For example, the knockout mutants of $G A M Y B$ in rice showed a severe defect in spikelet development, particularly in anther and pollen development (Kaneko et al. 2004).

GAMYB functions as a transcription factor, activating the expression of its downstream target genes regulating the exine and Ubisch body formation in pollen by directly binding to their promoters (Aya et al. 2009). Overexpression of GAMYB in barley (Hordeum vulgare) causes abnormal anthers with defective dehiscence, reduced length, and lighter color (Murray et al. 2003). In Arabidopsis, the GAMYB-like genes $M Y B 33$ and MYB65 contribute to anther development, similar to the role of $G A M Y B$ in rice and barley (Millar and Gubler 2005). These results demonstrate that GAMYB participates in GA signaling, which strongly affects floral development of angiosperms, mainly in anther and pollen growth.

The formation of male reproductive organs in plants requires numerous developmental steps including microspore maturation, meiosis, sporogenous cell differentiation, and stamen specification (McCormick 2004; Scott et al. 2004; Ma 2005; Guo and Liu 2012). Various investigations of the transcriptome identified many transcripts that are expressed in each developmental stage of anther and male gametophyte development in rice (reviewed by Guo and Liu 2012). Furthermore, some transcriptional analyses suggested that GA signal transduction occurs in developing microspores and the tapetum within the anther (Hirano et al. 2008; $\mathrm{Hu}$ et al. 2008). Microarray and comprehensive network analyses of the expressed genes in the rice anther have identified some genes in the GA signaling pathway, such as CYP703A3 and $K A R$, which encode a cytochrome P450 hydroxylase and $\beta$-ketoacyl reductase, respectively 
(Aya et al. 2009; Aya et al. 2011). This finding strongly suggests that GA is involved in the control of anther and pollen development (Kaneko et al. 2004; Chhun et al. 2007; Aya et al. 2009) and also indicates that GA is strongly associated with male sterility and spikelet fertility. Although recent investigations of GA signaling have focused on the consequences of GA deficiency in stamen development, over-activation of GA signaling also negatively affects silique fertility in Arabidopsis (Plackett et al. 2014).

In our previous study, we isolated heterogeneous inbred family-near isogenic lines (HNILs) from a $F_{7}$ recombinant inbred line (RIL) heterozygous for the functional EL1 allele and the nonfunctional el1 allele (Kwon et al. 2014). The natural missense mutation in el1 promotes flowering under non-inductive long-day conditions due to a defect in the protein kinase activity (Kwon et al. 2014, 2015). In this study, we found that the HNILs possessing EL1 or el1 showed different inflorescence and spikelet fertility phenotypes. Our results suggest that a loss of EL1/CKI activity causes excessive GA responses, resulting in abnormal development of male floral organs, finally resulting in decreased grain yield. Also, we discuss how the elite japonica cultivar Koshihikari, which harbors an el1 allele, could have normal GA responses and normal fertility.

\section{Results}

HNIL(H143) Plants Have Deformed Anther Structure And Decreased Pollen Viability

GA participates in various developmental processes in plants, including floral organ development (Cheng et al. 2004; Aya et al. 2009). EL1 phosphorylates SLR1, a major repressor of GA signaling (Dai and Xue 2010), and $\operatorname{slr} 1$ mutants are sterile (Ikeda et al. 2001). Previous studies reported that two types of natural missense mutations (types 2 and 3 ) in EL1 occur in the world rice collection and these mutations lead to compromised kinase activity of SLR1. HNIL(H143), harboring a type-2 el1 mutation derived from the 'H143' cultivar, shows an early-flowering phenotype only in long days, but not in short days, compared with the HNIL(M23), which has a functional EL1 allele derived from the 'Milyang23 (M23)' cultivar (Kwon et al. 2014).

While conducting flowering-time experiments, we observed a severe decrease in the seed setting rate of HNIL(H143) plants in field conditions. To explore the possible effects of the el1 mutation on rice fertility, we scrutinized the external and internal structure of spikelets of HNIL(M23) and HNIL(H143) plants at heading stage. The spikelets of HNIL(H143) showed normal structure (Fig. 1a and b), but the anthers of HNIL(H143) were shrunken and shorter than those of $\operatorname{HNIL}(\mathrm{M} 23)$,

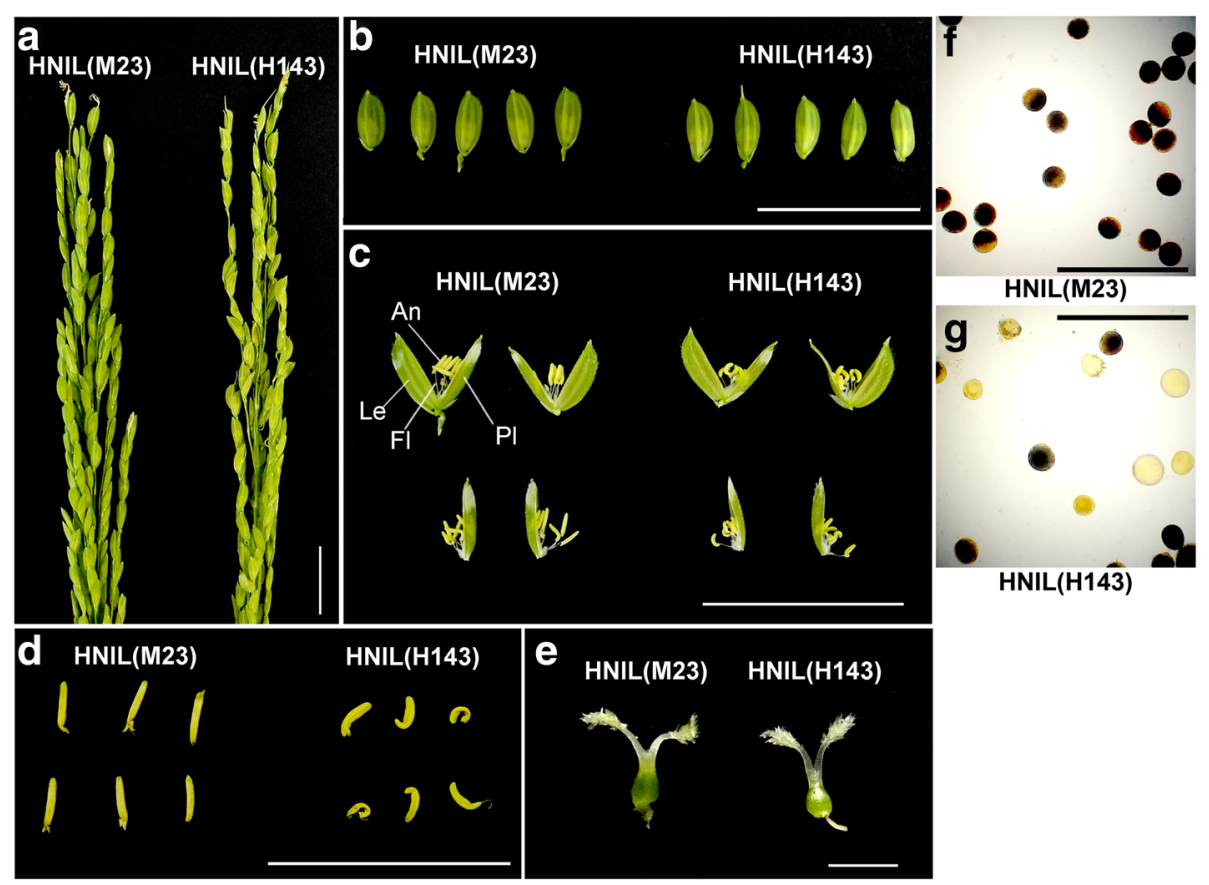

Fig. 1 HNIL(H143) showed abnormal anther and pollen development compared with HNIL(M23). a Panicle structure of HNIL(M23) and HNIL(H143) plants at the heading stage. Scale bar $=20 \mathrm{~mm}$. b Spikelets of the HNIL(M23) and HNIL(H143) plants. Scale bar $=20 \mathrm{~mm}$ (b and $\mathbf{c}$ ). $\mathbf{c}$ Flowers of the HNIL(M23) and HNIL(H143) plants. An, anther; Le, lemma; Fl, filament; Pl, palea. d Anthers of the HNIL(M23) and HNIL(H143) plants. Scale bar $=10$ mm. e Pistils of the HNIL(M23) and HNIL(H143) plants. Scale bar $=1 \mathrm{~mm}$. Pollen grains from (f) HNIL(M23) and (g) HNIL(H143) plants. Pollen stained with $\mathrm{I}_{2}-\mathrm{Kl}$ solution. Scale bar $=200 \mu \mathrm{m}(\mathbf{f}$ and $\mathbf{g})$. The data represent five independent biological replicates 
which showed normal structure (Fig. 1c and d). The pistils developed properly in the spikelets of HNIL(H143) (Fig. 1e). To examine the pollen viability in the shrunken anthers of HNIL(H143), we stained each anther using iodine-potassium iodide $\left(\mathrm{I}_{2}-\mathrm{KI}\right)$ solution, which revealed that HNIL(H143) pollen had lower viability than HNIL(M23) pollen (Fig. If and g). We also found that the pollen viability of the T-DNA insertion el1 knockout mutant [hereafter $\mathrm{ZH} 11($ el1)] was also reduced compared to its parental cultivar, 'Zhonghua11 (ZH11)' (Additional file 1: Figure S1). Finally, we examined the spikelets of 'H143' and 'M23', which are the parental cultivars for the HNILs (Additional file 2: Figure S2). We also found that the H143 plants have shrunken anthers, similar to HNIL(H143) (Additional file 2: Figure S2). The H143 plants had fewer mature pollen grains than the M23 plants and many of the pollen grains in H143 were poorly stained by $\mathrm{I}_{2}-\mathrm{KI}$ solution (Additional file 2 : Figure S2). Taken together, these results suggest that the shrunken anther structure and reduced pollen viability of HNIL(H143) are caused by the el1 mutation in $\mathrm{H} 143$.

\section{HNIL(H143) Spikelets Showed Up-Regulated Expression Of GAMYB And Genes Related To Exine Formation At The Heading Stage}

SLR1, phosphorylated and activated by EL1/CKI, represses the expression of the GA-responsive gene $G A M Y B$, which encodes a transcription factor that positively regulates the $\alpha$-amylase gene in aleurone cells (Itoh et al. 2002; Ueguchi-Tanaka et al. 2007b; Dai and Xue 2010). Loss of SLR1 function in rice induces a sterile phenotype, like mutants of its orthologue slender 1 $(\sin 1)$ in barley (Lanahan and Ho 1988; Ikeda et al. 2001). GAMYB is also closely associated with anther development, acting through GA signaling to regulate several genes related to exine formation (Kaneko et al. 2004; Aya et al. 2009). In particular, transgenic barley plants overexpressing $H \nu G A M Y B$ showed a reduction in anther size and increased male sterility (Murray et al. 2003).

We examined the transcript levels of GAMYB in the spikelets in HNIL(M23) and HNIL(H143) at heading stage. We found that GAMYB expression was upregulated in HNIL(H143) compared to HNIL(M23) (Fig. 2a). Moreover, transcript levels of the exine formation-related genes CYP703A3 and KAR, which are activated by GAMYB, were also up-regulated in HNIL(H143) (Fig. 2b and c). This result strongly suggests that abnormal up-regulation of the three GA signalingrelated genes may cause the deformity of anthers in HNIL(H143).

\section{HNIL(H143) Plants Showed Over-Activation Of GA Signaling} EL1/CKI phosphorylates and stabilizes SLR1, which decreases the response to GA signaling. Thus, ZH11(el1) plants exhibit increased GA signaling response, even in the absence of GA or in the presence of uniconazole, an inhibitor of GA biosynthesis, or ABA; the el1 seeds also display much higher $\alpha$-amylase activity (Dai and Xue 2010).

For the functional analysis of the natural el1 mutant allele in these HNILs (type 2; Kwon et al. 2014), we examined the $\alpha$-amylase activity of mature seeds of HNIL(M23) and HNIL(H143), and their parents M23 and H143. Compared with HNIL(M23), the imbibed seeds of HNIL(H143) had remarkably elevated $\alpha$ amylase activity in the absence of GA; the inhibitory effect of ABA on the $\alpha$-amylase activity was also significantly alleviated (Fig. 3a and Additional file 3: Figure S3). The seeds of H143 showed a similar, enhanced $\alpha$-amylase activity, compared with M23 (Fig. 3b and Additional file 3: Figure S3). Following treatment with exogenous $\mathrm{GA}_{3}$,
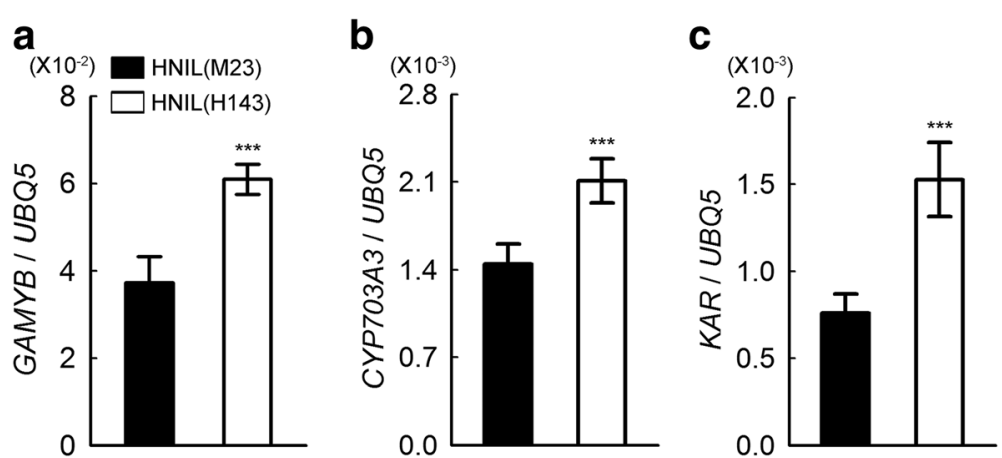

Fig. 2 Up-regulation of GAMYB and pollen formation-related genes in the spikelets of HNIL(H143). Relative transcript levels of GAMYB (a), CYP703A3 (Cytochrome P450 hydroxylase) (b), KAR (B-ketoacyl reductase) (c) in HNIL(M23) and HNIL(H143) spikelets were normalized to the transcript levels of UBQ5. The RT-qPCR was performed with total RNA from spikelets at heading stage. The data were obtained from three independent biological replicates. Reactions were repeated at least twice. Student's $t$-test was used for statistical analysis $\left({ }^{* *} P<0.001\right)$. Means and standard deviations are recorded as values and vertical bars, respectively 

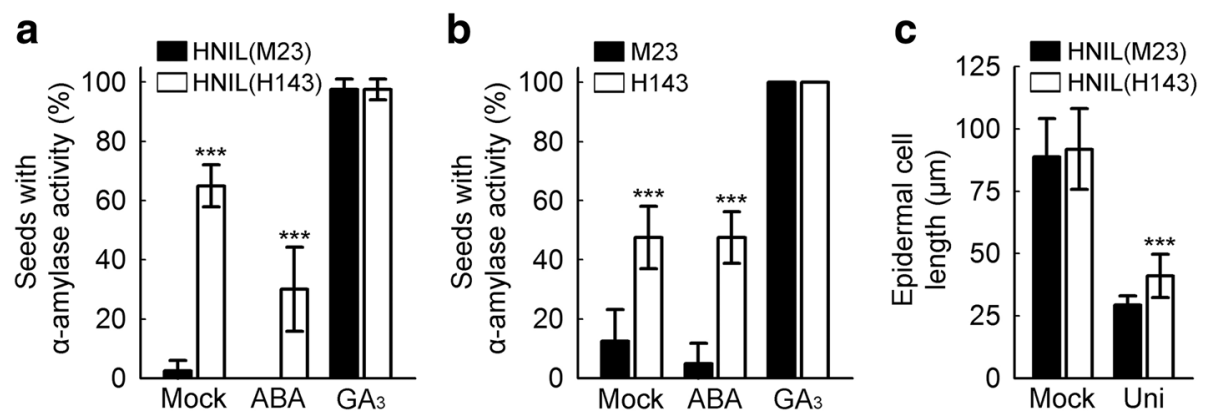

Fig. $3 \mathrm{GA}$ responses were enhanced in the seeds carrying the ell(H143) allele. Assays of a-amylase activity in the seeds of HNIL(M23) and $\mathrm{HNIL}(\mathrm{H} 143)$ (a) and M23 and H143 (b) on plates containing starch. The seeds were incubated on plates containing ABA (10 $\mu \mathrm{M})$ or GA 3 (1 mM) for 3 days at $28^{\circ} \mathrm{C}$. The numbers of seeds with a-amylase activity were compared by Student's $t$-test $\left(*^{* *} P<0.001, n=40\right)$. This experiment was repeated at least 3 times. $\mathbf{c}$ Length of the epidermal cells in the 2 nd leaf sheath of HNIL(M23) and HNIL(H143) plants. The plants were treated with uniconazole (Uni; GA biosynthesis inhibitor) for 1 day and grown in a growth chamber for 7 days. Student's $t$-test was used for statistical analysis $\left({ }^{* *} P<0.001, \mathrm{n}=10\right)$

almost $100 \%$ of the seeds of both genotypes induced $\alpha$-amylase activity (Fig. 3a and b).

GA stimulates the internode elongation and plant height. ZH11(el1) also had much longer epidermal cells in the leaf sheath than WT after uniconazole treatment, likely resulting from the increased GA response in ZH11(el1) plants (Dai and Xue 2010). We also observed that the epidermal cells of the 2nd leaf sheath in HNIL(H143) were significantly longer than those in HNIL(M23) after uniconazole treatment (Fig. 3c), supporting the notion that the ell mutation (type 2) in H143 leads to an enhanced GA signaling response, even in the absence of GA.

\section{Loss Of EL1 Function Decreased Yield-Related Traits By Reducing Spikelet Fertility}

To examine the effect of the el1 mutation in H143 on grain production, we measured yield and yield components in HNIL(M23) and HNIL(H143) plants grown under natural long days in the paddy field in 2012 and 2013 (Fig. 4 and Additional file 4: Figure S4). We measured main panicle length, number of panicles per plant, number of spikelets per main panicle, 500-grain weight, grain yield per plant, and seed setting rate. Among these six agronomic traits, three major traits (500-grain weight, yield per plant, and seed setting rate) were significantly reduced in HNIL(H143) (Fig. 4d and f) and the others did not show any difference between HNIL(M23) and HNIL(H143), although the panicle number per plant in HNIL(H143) was slightly higher (Fig. 4a-c). The decrease in these three yield-related traits in $\mathrm{HNIL}(\mathrm{H} 143)$ was observed in both years (Additional file 4: Figure S4), suggesting that dramatic reduction of seed setting rate in HNIL(H143) is the main reason for the reduction of grain yield. This indicates that EL1 function is essential for sustaining grain production, in addition to delaying flowering time in non-inductive long days (Kwon et al. 2014).
Next, we examined the yield and yield components of H143 and M23, the parental cultivars of HNIL(M23) and HNIL(H143) (Additional file 5: Figure S5). Most yieldrelated traits were lower in H143 than in M23, except the number of panicles per plant. Consistent with the disparity of seed setting rate in HNIL(M23) and HNIL(H143), the spikelet fertility of H143 was much lower than that of M23, which appears to be a main reason for the decrease in yield per plant. Together, these data suggest that the reduced fertility in HNIL(H143) was influenced by the natural allele of el1 inherited from H143.

Finally, we examined the yield-related traits of ZH11(el1) corresponding to HNIL(H143) by comparing with those of its parental cultivar ZH11 corresponding to HNIL(M23) (Additional file 6: Figure S6). This comparison revealed that the seed setting rate of $\mathrm{ZH} 11(e l 1)$ was about $12 \%$ lower than that of ZH11 (Additional file 6: Figure S6). Taking all of these results together, we concluded that EL1 is essential for high yield potential, especially for maintaining spikelet fertility.

\section{Koshihikari, An Elite Japonica Cultivar Carrying An el1 Mutation, Has No Defect In GA Signaling Or Spikelet Fertility}

We previously reported that two types of missense mutations in the highly conserved serine/threonine kinase domain of EL1 exist in the japonica cultivars, H143 (type 2) and Koshihikari (type 3) (Kwon et al. 2014). Both natural mutations in EL1 cause a loss of kinase activity, leading to a failure in phosphorylation of SLR1 (Dai and Xue 2010; Kwon et al. 2014). Consistent with the el1 mutant phenotype, Koshihikari does show early flowering under long-day conditions (Matsubara et al. 2008; Hori et al. 2013; Kwon et al. 2014). Thus, we expected that Koshihikari would exhibit a similar defect in GA signaling and a low seed setting rate. However, Koshihikari showed no significant difference in the yield 

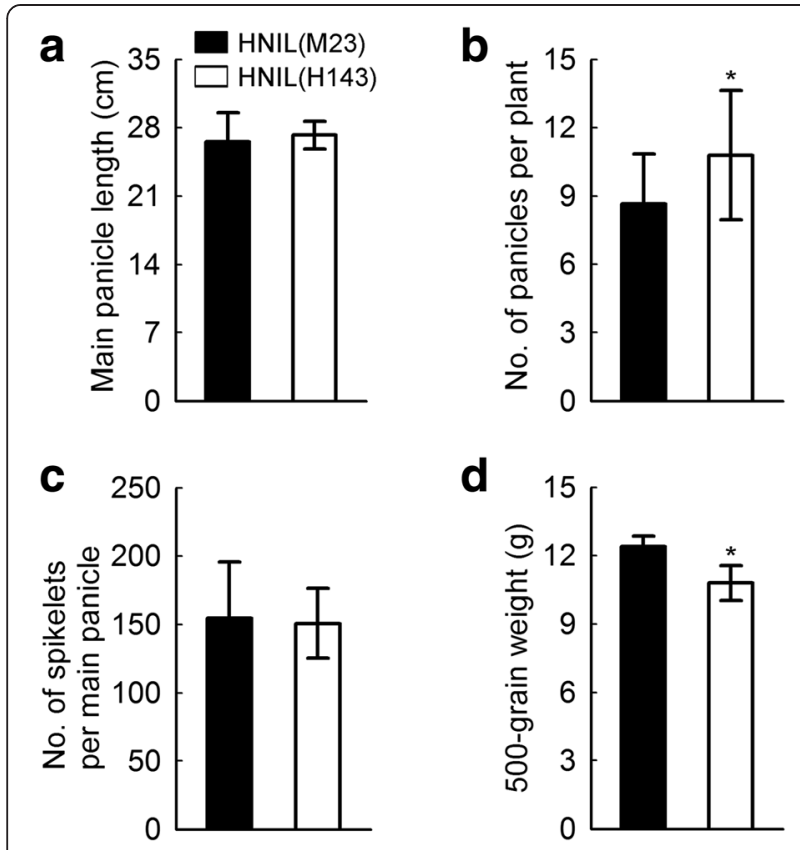

e

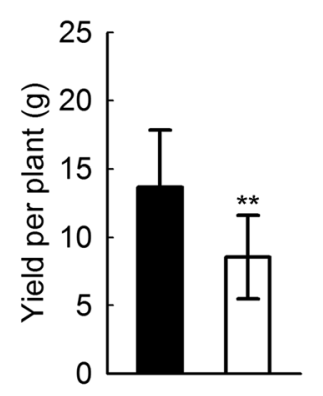

\section{f}

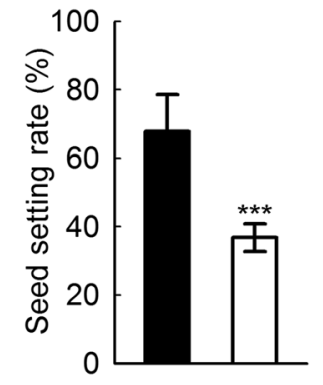

g

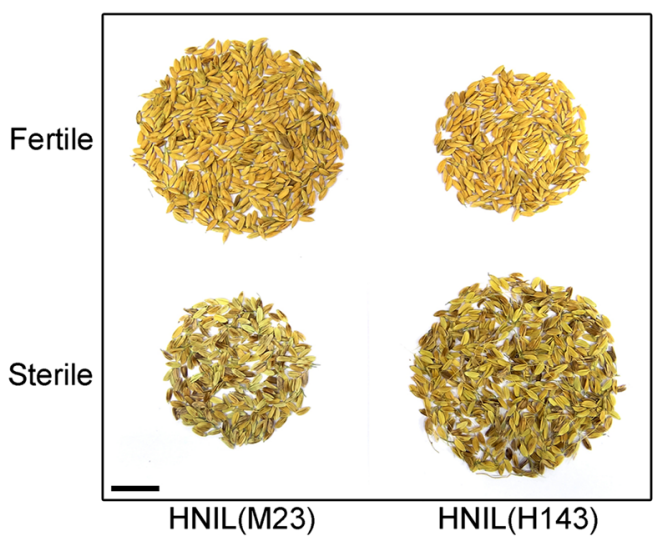

Fig. 4 Yield-related agronomic traits were remarkably lower in HNIL(H143) compared with HNIL(M23). Agronomic traits were measured in HNIL(M23) and HNIL(H143) grown under natural long days in 2013.

The measured traits were: main panicle length (a), number of panicles per plant (b), number of spikelets per main panicle (c), 500-grain weight (d), yield per plant (e), and seed setting rate (f). Twenty plants were used to measure each trait. Student's $t$-test was used for statistical analysis ( $\left.{ }^{*} P<0.05,{ }^{* *} P<0.01,{ }^{* * *} P<0.001\right)$. Means and standard deviations are marked as values and vertical bars, respectively. (g) Fertile and sterile seeds from the whole plants of HNIL(M23) and HNIL(H143). Scale bar $=2 \mathrm{~cm}$ and yield-related components compared with the highyield japonica cultivar 'Dongjin (type 1; functional EL1)' or the high-yield indica cultivar 'Milyang23 (type 4; functional EL1)' (Kwon et al. 2014; Table 1). In particular, the seed setting rate of Koshihikari was close to $90 \%$, suggesting no negative effect of the ell mutation on anther development or spikelet fertility in Koshihikari. We also found by observation of $\alpha$-amylase activity that the response to GA was not altered at all in the seeds of Koshihikari compared to that of the elite japonica cultivar Nipponbare (Fig. 5). Moreover, the transcript levels of GAMYB showed no significant increase in the germinating seeds of Koshihikari, in contrast to the high GAMYB transcript levels in ZH11(el1) and HNIL(H143) (Fig. 6).

\section{Discussion}

\section{EL1 Is Essential For Anther Development}

Many reports have shown that GA signaling affects floral organ development, since defects in GA signaling often result in male sterility; indeed, several GAinsensitive or GA-deficient mutants exhibit anther deformities and/or produce sterile flowers in rice, petunia, maize, tomato, barley, and Arabidopsis (Lanahan and Ho 1988; Nester and Zeevaart 1988; Jacobsen and Olszewski 1991; Evans and Poethig 1995; Goto and Pharis 1999; Ikeda et al. 2001; Izhaki et al. 2002; Cheng et al. 2004; Kaneko et al. 2004; Chhun et al. 2007). Moreover, some factors in the GA signaling cascade affect flower development, particularly anther formation in rice (Kaneko et al. 2004; Chhun et al. 2007; Aya et al. 2009).

EL1/CKI has a significant role in alleviating the response to GA signaling by phosphorylating the DELLA protein SLR1, a major repressor of GA signaling in rice (Dai and Xue 2010). In ZH11(el1) plants, the nonphosphorylated SLR1 is degraded rapidly, suggesting that the phosphorylation of SLR1 by EL1 is essential for SLR1 stability (Dai and Xue 2010). The failure of posttranslational modification of SLR1 protein triggered derepression of its downstream genes, including GAMYB, in $\mathrm{HNIL}(\mathrm{H} 143)$ (Fig. 2), leading to an increase in responses downstream of GA signaling, such as $\alpha$-amylase activity in the endosperm of germinating seeds (Fig. 3 and Additional file 3: Figure S3).

The GA signaling transducer GAMYB activates expression of $\alpha$-amylase in the aleurone cells of cereal grains by directly binding to the promoter of the gene encoding $\alpha$-amylase (Gubler et al. 1995; Gubler et al. 1999; Gubler et al. 2002; Aya et al. 2009). GAMYB also participates in anther development and acts as a transcription factor by directly binding to the promoters of several GA-regulated genes that are responsible for exine and Ubisch body formation (Kaneko et al. 2004; Aya 
Table 1 Agronomic traits of three rice cultivars in Korea and Japan

\begin{tabular}{lllllll}
\hline Cultivar & $\begin{array}{l}\text { Main panicle } \\
\text { length }(\mathrm{cm})\end{array}$ & $\begin{array}{l}\text { No. of } \\
\text { panicles/plant }\end{array}$ & $\begin{array}{l}\text { No. of spikelets/main } \\
\text { panicle }\end{array}$ & $\begin{array}{l}\text { 500-seed } \\
\text { weight }(\mathrm{g})\end{array}$ & $\begin{array}{l}\text { Seed setting } \\
\text { rate (\%) }\end{array}$ & $\begin{array}{l}\text { Yield/plant (g) } \\
\text { Dongjin (j) }\end{array}$ \\
\hline $20.2 \pm 0.8$ & $9.4 \pm 1.7$ & $124.6 \pm 12.6$ & $12.6 \pm 0.9$ & $93.6 \pm 1.7$ & $23.4 \pm 4.3$ \\
Milyang23 (i) & $25.8 \pm 1.7$ & $9.8 \pm 1.7$ & $186.7 \pm 27.4$ & $11.4 \pm 0.8$ & $84.9 \pm 1.8$ & $35.5 \pm 4.7$ \\
Koshihikari (j) & $19.7 \pm 0.9$ & $12.6 \pm 2.5$ & $121.9 \pm 12.8$ & $11.0 \pm 1.1$ & $88.8 \pm 2.2$ & $25.3 \pm 3.2$ \\
\hline
\end{tabular}

Twenty plants were grown in Suwon, Korea $\left(37^{\circ} \mathrm{N}\right)$ in 2014 . The (j) and (i) indicate japonica and indica, respectively

et al. 2009). The gamyb mutants show defects in floral organs, especially in anther development (Kaneko et al. 2004), because the expression of CYP703A3 and $K A R$, which are required for normal development of anther and pollen, was dramatically suppressed in gamyb mutants (Aya et al. 2009). Paradoxically, overexpression of $G A M Y B$ in barley also causes defects in anther development, such as impaired dehiscence, decreased anther length, and lighter color (Murray et al. 2003), similar to the phenotype of gamyb mutants.

In this study, we showed that the transcript levels of $G A M Y B$ increased significantly in $\mathrm{HNIL}(\mathrm{H} 143)$, possibly due to a lack of SLR1 activity caused by the el1 mutation (Fig. 2); this may cause the abnormal development of anthers observed in el1 mutants (Fig. 1). These results suggest that normal anther development requires the precise spatiotemporal control of GAMYB transcription. Although further study is needed, our present results provide evidence that the alteration in the response to GA caused by the el1 mutation impairs anther development, in addition to causing early flowering under non-inductive long-day conditions in temperate and cooler regions (Kwon et al. 2014).

\section{Mutation Of EL1 Negatively Affects Spikelet Fertility}

Plants that have excessive GA-induced signaling show decreased pollen viability and a sterile phenotype. For example, transgenic barley overexpressing $H \nu G A M Y B$ showed decreased spikelet fertility, and thus a severe reduction in grain yield (Murray et al. 2003). The rice gamyb null mutants had fewer spikelets per panicle and a defect in the induction of $\alpha$-amylase expression in response to GA treatment (Kaneko et al. 2004). Similarly, the loss-of-function $\operatorname{slr1-1}$ and the gain-of-function Slr1d3 mutants exhibit sterile and semi-fertile phenotypes, respectively, in rice (Ikeda et al. 2001; Chhun et al. 2007). Although Slr1-d3 mutants produced normal floral organs with morphologically normal pistils and stamens, they showed low pollen viability compared to wild type, leading to semi-fertility in the Slr1-d3 mutants (Chhun et al. 2007).

Our further phenotypic characterization showed that many agronomic traits related to yield and yield components were lower in $\operatorname{HNIL}(\mathrm{H} 143)$ compared with the EL1 HNIL(M23). Especially, the seed setting rate was significantly reduced, leading to a reduction of grain yield in HNIL(H143), suggesting that the HNIL(H143)

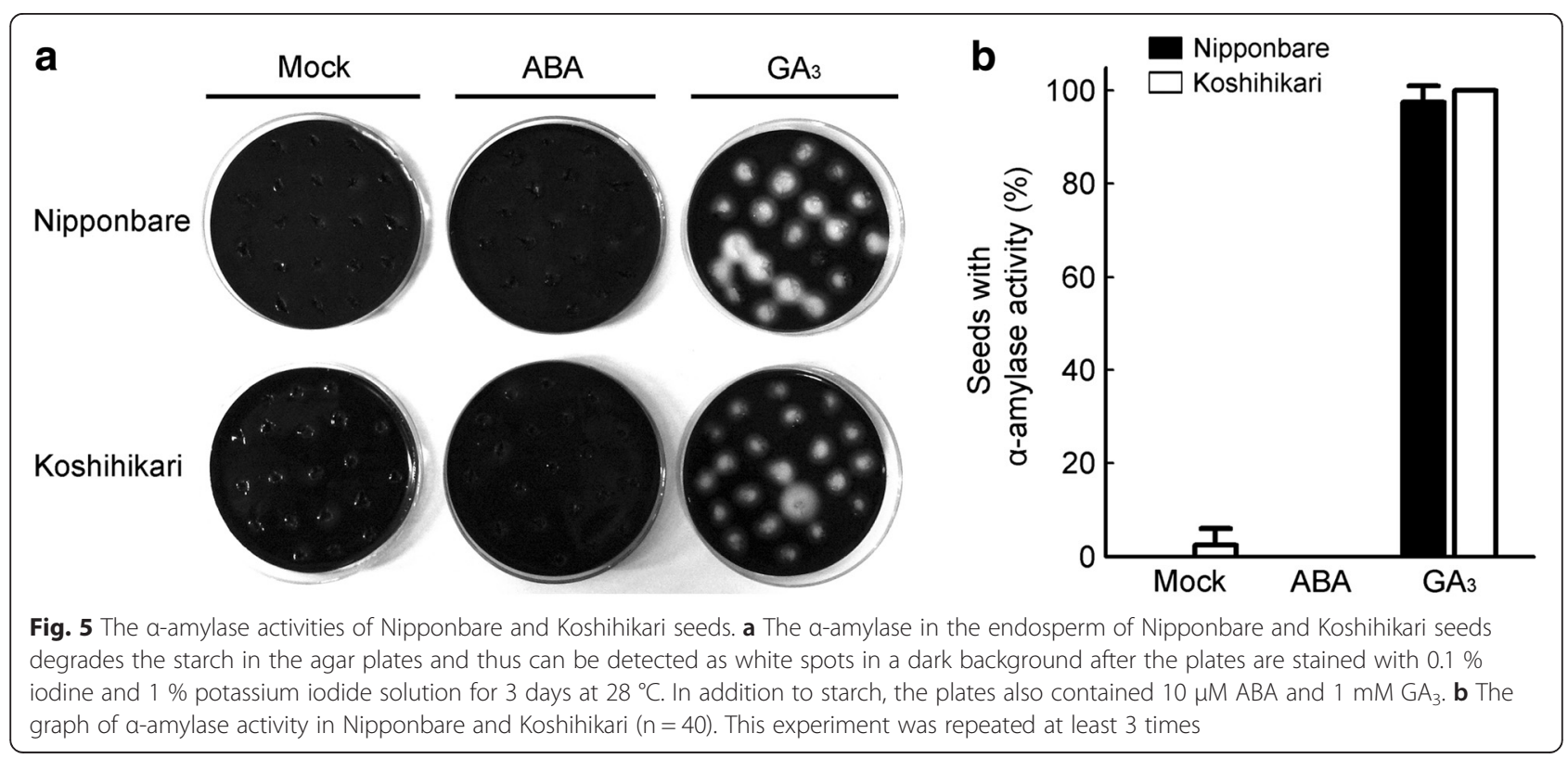



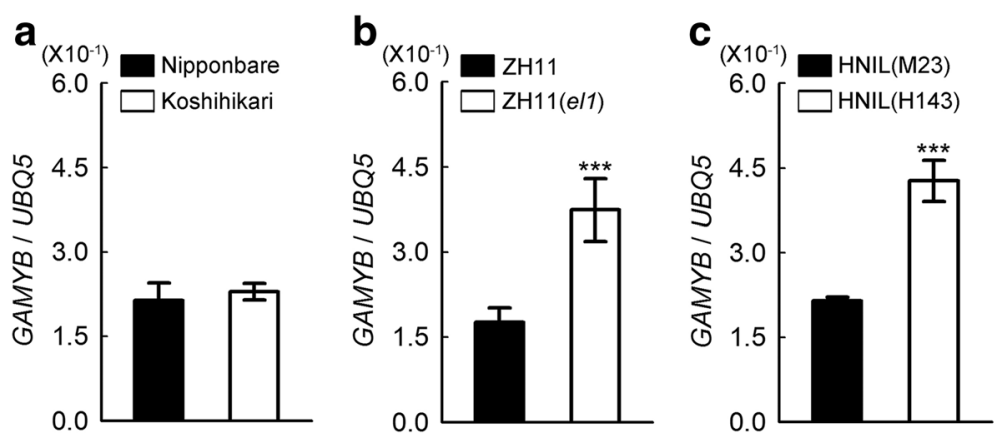

Fig. 6 The levels of GAMYB transcript were not upregulated in Koshihikari. Relative transcript levels of GAMYB in Nipponbare and Koshihikari (a), ZH11 and ZH11(e/1) (b), and HNIL(M23) and HNIL(H143) (c), were normalized to the transcript levels of UBQ5. The RT-qPCR was performed with total RNA from germinating seeds 2 days after soaking. Means and standard deviations were obtained from three replications. The experiments were repeated twice with similar results and Student's $t$-test was used for statistical analysis $\left({ }^{* *} P<0.001\right)$

el1 allele affects yield and yield components (Fig. 4). The decrease of seed setting rate was also found in H143 and ZH11(el1), indicating that the el1 mutation negatively affects spikelet fertility, because of low pollen viability (Additional file 1: Figure S1, Additional file 2: Figure S2, Additional file 5: Figure S5 and Additional file 6: Figure S6). Taken together, these results indicate that a loss of function in EL1/CKI decreases SLR1 activity and stability, inducing a constitutive GA response like the rice slr1-1 mutants (Ikeda et al. 2001; Murray et al. 2003; Dai and Xue 2010). Therefore, it is considered that excessive GA signaling caused by the el1 mutation negatively affects seed setting rate.

\section{No Defect Of GA Signaling In Koshihikari Carrying The el1 (Type 3) Null Allele}

We previously reported that naturally occurring ell alleles (types 2 and 3) contribute to rice adaptation to growth in the northernmost, cooler regions by inducing photoperiod insensitivity and early flowering under long-day conditions (Kwon et al. 2014). In addition to the positive effect of el1 in rice domestication and adaptation, this study also reports its negative effect on spikelet fertility. However, by investigating the response to GA signaling and yield-related traits of Koshihikari plants, which carry a nonfunctional el1 (type 3) allele (Hori et al. 2013; Kwon et al. 2014), we found that the Koshihikari cultivar shows a normal GA response and seed setting rate, compared with other elite japonica cultivars possessing a functional EL1 allele (Fig. 5 and Table 1). In addition, GAMYB expression in Koshihikari was almost the same as that of Nipponbare, ZH11, and HNIL(M23), in contrast with the higher GAMYB expression observed in ZH11(el1) and HNIL(H143) (Fig. 6). This finding strongly suggests that GAMYB expression and/or GA signaling is controlled normally in Koshihikari, although the el1 (type 3) mutation contributes to the early flowering of Koshihikari under long-day conditions in temperate regions. In Arabidopsis, mutants for all five DELLA paralogs still show basal levels of fertility in the Landsberg erecta (Ler) ecotype, indicating that DELLA activity in Ler is not essential to maintain fertility (Dill and Sun 2001; Fuentes et al. 2012). In contrast, the rga gai mutants in the Columbia (Col-0) ecotype show male sterility induced by post-meiotic defects in pollen development, and the sterile phenotype are recovered by transgenic introduction of DELLAs into developing pollen (Plackett et al. 2014). Furthermore, the distinct fertility phenotype of Col-0 and Ler can be caused by either differences in downstream signaling mechanisms or altered expression of DELLA proteins (Plackett et al. 2014). Thus, we propose that the introduction or natural variation of other unknown regulator(s) offsetting excessive GA signaling may have occurred during the breeding of Koshihikari. These unknown regulators act downstream of SLR1 in GA signaling, or directly in SLR1 protein modification and stability (Fig. 7). Therefore, further studies to identify the unknown factor(s) are ongoing to explain how Koshihikari can achieve normal GA signaling and thus maintain spikelet fertility in the el1 mutant background.

\section{Pleiotropy Of The el1 Mutation In Rice Development}

Two recent reports showed the molecular mechanism of EL1 function in the photoperiodic pathway in rice flowering (Hori et al. 2013; Kwon et al. 2014). In addition, it had been published that EL1 functions in GA signaling by demonstrating the in vitro phosphorylation of SLR1 (Dai and Xue 2010). EL1/CKI acts as a flowering repressor by interacting with and directly phosphorylating Ghd7 and OsPRR37; this modification delays heading date in non-inductive long-day conditions (Hori et al. 2013; Kwon et al. 2015). This suggests that the targets downstream of EL1/CKI act throughout various genetic and signaling pathways during rice development, such as 


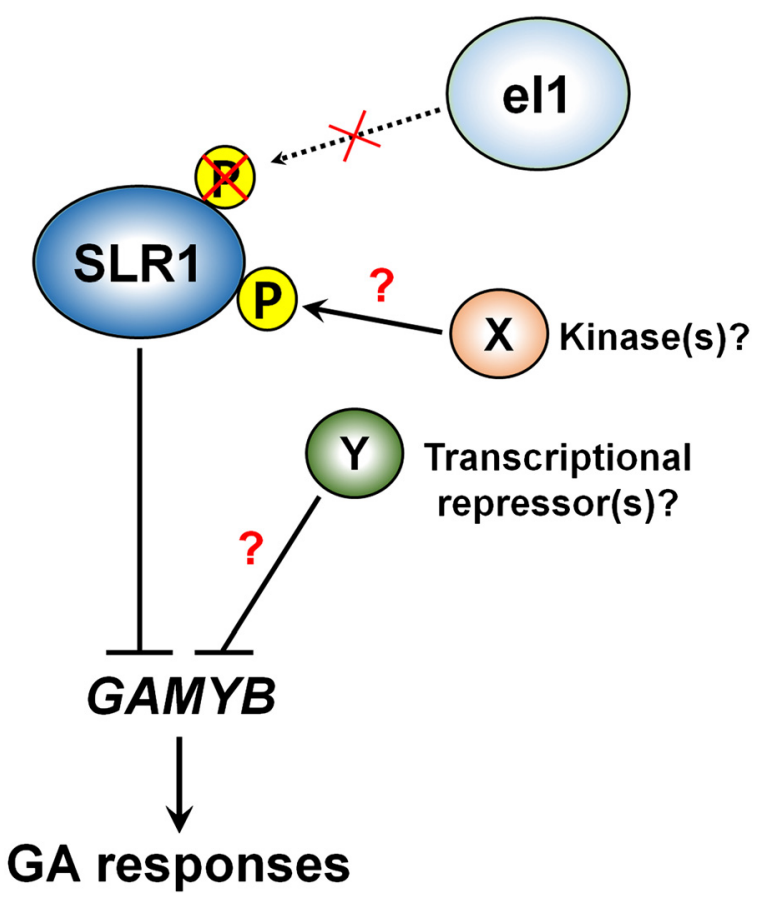

Fig. 7 A schematic model of the GA response in Koshihikari. In the Japanese elite japonica cultivar 'Koshihikari', which carries an el1 mutation, the phosphorylation of SLR1 by EL1 is impaired. We speculate that GAMYB transcription and GA responses are normally regulated by an as-yet-unidentified kinase or kinases ( $X$ ) that phosphorylate SLR1 or repressor(s) of GAMYB expression ( $Y$ ) to produce the high spikelet fertility observed in Koshihikari. P, phosphorus. Arrow and blocked lines indicate positive and negative regulation, respectively

inflorescence formation, flowering, stem elongation, and germination. Moreover, the el1 mutant displays other phenotypes related to GA action, such as taller plants and longer epidermal cells (Dai and Xue 2010). In this study, we demonstrate that the el1 mutation decreases grain yield because of a reduction of seed setting rate.

\section{Conclusion}

Although the el1 mutation contributes to early flowering of japonica rice cultivars under non-inductive long-day conditions in temperate and cooler regions, it can also negatively affect grain production. The enhanced response to GA signaling in the rice el1 mutants induces overexpression of $G A M Y B$ and the pollen formation-related genes $C Y P 703 A 3$ and $K A R$ during spikelet development, causing defects in anther development and pollen viability. However, the elite japonica cultivar Koshihikari, harboring an el1 allele, appears to overcome this disadvantage by having unknown regulator(s) that maintain normal GA signaling. This may explain why the el1 allele occurs in only a few extremely early-flowering japonica cultivars in the world rice collections (Kwon et al. 2014).

\section{Methods}

Plant Materials And Growth Conditions

Heterogeneous inbred family-near isogenic lines (HNILs) derived from crosses between a japonica rice 'H143' and a japonica-indica hybrid rice 'M23' were used in this study (Koo et al. 2013; Kwon et al. 2014). The EL1 and el1 alleles of HNIL(M23) and HNIL(H143) were derived from M23 and H143, respectively (Kwon et al. 2014). H143, M23, ZH11, and ZH11(el1) (Dai and Xue 2010) were grown for observation of spikelet structure and analysis of agronomic traits. The plants for this study were grown in the paddy field of Seoul National University experimental farm in Suwon, Korea $\left(37^{\circ} \mathrm{N}\right.$ latitude) from April to October. The day lengths of Suwon were about $13.5 \mathrm{~h}$ (April), $14.2 \mathrm{~h}$ (May), $14.7 \mathrm{~h}$ (June), $14.3 \mathrm{~h}$ (July) and $13.6 \mathrm{~h}$ (August). Cultivation of these plants followed standard agricultural methods.

\section{Reverse Transcription And Quantitative Real-Time PCR (RT-qPCR)}

Total RNA from spikelets or germinating seeds was extracted using the Total RNA Extraction Kit (Macrogen, Korea). First-strand cDNA was synthesized from $2 \mu \mathrm{g}$ total RNA using oligo $(\mathrm{dT})_{15}$ primer and M-MLV reverse transcriptase (Promega, Madison, WI, USA). Transcript levels of GA signaling-related genes were detected by qPCR using gene-specific primers and Ubiquitin5 (UBQ5) as the internal control (Additional file 7: Table S1). Reactions of $20 \mu \mathrm{l}$ included $2 \mu \mathrm{l}$ of $0.5 \mu \mathrm{M}$ primer, $2 \mu \mathrm{l}$ of cDNA mixture, and $10 \mu \mathrm{l}$ of $2 \mathrm{X}$ QuantiTect LightCycler 480 SYBR Green I Master Mix (Roche, Basel, Switzerland). qPCR was performed with the Light Cycler 2.0 instrument (Roche) using the program: $95{ }^{\circ} \mathrm{C}$ for $2 \mathrm{~min}, 45$ cycles of $95^{\circ} \mathrm{C}$ for $10 \mathrm{~s}, 59^{\circ} \mathrm{C}$ for $10 \mathrm{~s}$, and $72{ }^{\circ} \mathrm{C}$ for $10 \mathrm{~s}$.

\section{a-Amylase Assay}

The $\alpha$-amylase assay was performed as previously described (Yamaguchi 1998; Dai and Xue 2010). Briefly, seed coats and embryos were removed and only endosperms were sterilized in $1 \% \mathrm{NaClO}$ containing $0.01 \%$ TWEEN 20 for $30 \mathrm{~min}$, and then rinsed with distilled water 6 times. The half-seeds were placed on $2 \%$ phytoagar medium containing $0.2 \%$ soluble starch, and media including $\mathrm{GA}_{3}$ or ABA were used for analysis. All the plates were incubated in the dark at $28^{\circ} \mathrm{C}$ for 3 days. After that, the endosperms were removed from the plates, and then the plates were stained with $0.1 \%$ iodine and $1 \%$ potassium iodide. Seeds with $\alpha$-amylase activity were quantified by counting white spots.

\section{Pollen Viability Analysis}

To determine pollen viability, six anthers were picked from a spikelet at heading stage and moved to a glass slide. The anthers were broken and stained with $0.1 \%$ 
iodine and $1 \%$ potassium iodide solution $\left(\mathrm{I}_{2}-\mathrm{KI}\right)$. Five minutes later, the pollen grains were observed using an optical microscope (Olympus, BX50). Viable pollen grains were identified by their black color and round shape; sterile pollen grains were identified by their yellow color and shrunken shape.

\section{Additional Files}

Additional file 1: Figure S1. Flower and pollen structure of $\mathrm{ZH} 11$ and ZH11(e/1) plants. (A) Panicle structure of $\mathrm{ZH} 11$ and $\mathrm{ZH} 11$ (e/1) at the heading stage. Scale bar $=20 \mathrm{~mm}$. (B) Spikelets of ZH11 and ZH11(e/1). Scale bar $=20 \mathrm{~mm}$. (C) Flowers of the ZH11 and ZH11(e/1) plants. An, anther; Le, lemma; Fl, filament; Pl, palea. Scale bar $=20 \mathrm{~mm}$. (D) Anthers of the $\mathrm{ZH} 11$ and $\mathrm{ZH} 11$ (e/1) plants. Scale bar $=10 \mathrm{~mm}$. (E) Pistils of the ZH11 and ZH11(e/1) plants. Scale bar $=1 \mathrm{~mm}$. The pollen grains from (F) $\mathrm{ZH} 11$ and $(\mathbf{G}) \mathrm{ZH} 11$ (e/1) plants. Pollen was stained with $\mathrm{I}_{2}-\mathrm{Kl}$ solution. Scale bar $=200 \mu \mathrm{m}$ (F and $\mathbf{G})$. The data represent five independent biological replicates. (DOCX $1785 \mathrm{~kb}$ )

Additional file 2: Figure S2. Flower and pollen structure of $\mathrm{H} 143$ and M23. (A) Panicle structure of M23 and the $\mathrm{H} 143$ at the heading stage. Scale bar $=20 \mathrm{~mm}$. (B) Spikelets of the M23 and H143 plants. Scale bar $=20 \mathrm{~mm}$. (C) Flowers of the M23 and H143 plants. An, anther; Le, lemma; Fl, filament; Pl, palea. Scale bar $=20 \mathrm{~mm}$. (D) Anthers of the M23 and $\mathrm{H} 143$ plants. Scale bar $=10 \mathrm{~mm}$. (E) Pistils of the M23 and H143 plants. Scale bar $=1 \mathrm{~mm}$. Pollen grains from (F) M23 and (G) H143 plants. Pollen stained with $\mathrm{I}_{2}-\mathrm{KI}$ solution. Scale bar $=200 \mu \mathrm{m}(\mathbf{F}$ and $\mathbf{G})$. The data represent five independent biological replicates. (DOCX $1774 \mathrm{~kb}$ )

Additional file 3: Figure S3. Assay for a-amylase activity on starch plates. Production of a-amylase from the endosperm of seeds was detected as white spots. The starch plates also contained $10 \mu \mathrm{M}$ ABA or $1 \mathrm{mM} \mathrm{GA}_{3}$. The plates were stained with $0.1 \%$ iodine and $1 \%$ potassium iodide solution. (A) HNIL(M23) and HNIL(H143) seeds; (B), M23 and H143 seeds. These experiments were repeated at least three times. (DOCX $1006 \mathrm{~kb}$ )

Additional file 4: Figure S4. Yield-related agronomic traits of HNIL(M23) and HNIL(H143) ( $\left.F_{7: 11}\right)$. Agronomic traits were measured in HNIL(M23) and HNIL(H143) plants grown under natural long days in 2012. The measured traits were: $(\mathbf{A})$ main panicle length, (B) number of panicles per plant, (C) number of spikelets per main panicle, (D) 500-grain weight, (E) yield per plant, and (F) seed setting rate. 20 plants were used to measure each trait. Student's $t$-test was used for statistical analysis ( $P<0.05$, $\left.{ }^{* *} P<0.01,{ }^{* * *} P<0.001\right)$. Means and standard deviations are marked as values and vertical bars, respectively. (G) Fertile and sterile seeds from the whole plants of HNIL(M23) and HNIL(H143). Scale bar $=2 \mathrm{~cm}$. (DOCX $1896 \mathrm{~kb})$

Additional file 5: Figure S5. Yield-related agronomic traits of M23 and $\mathrm{H} 143$. Agronomic traits were measured in M23 and H143 plants grown under natural long days in 2013. The measured traits were: $(\mathbf{A})$ main panicle length, (B) number of panicles per plant, (C) number of spikelets per main panicle, (D) 500-grain weight, (E) yield per plant and (F) seed setting rate. 20 plants were used to measure each trait. Student's $t$-test was used for statistical analysis $\left({ }^{*} P<0.05,{ }^{*} P<0.01,{ }^{*}{ }^{*} P<0.001\right)$. Means and standard deviations are marked as values and vertical bars, respectively. (G) Fertile and sterile seeds from the whole plants of M23 and H143. Scale bar $=2 \mathrm{~cm}$. (DOCX $1639 \mathrm{~kb}$ )

Additional file 6: Figure S6. Yield-related agronomic traits of $\mathrm{ZH} 11$ and $\mathrm{ZH} 11(\mathrm{e} / 1)$. Agronomic traits were measured in $\mathrm{ZH} 11$ and $\mathrm{ZH} 11(\mathrm{e} / 1)$ grown under natural long days in 2014. The measured traits were: $(\mathbf{A})$ main panicle length, (B) number of panicles per plant, $(\mathbf{C})$ number of spikelets per main panicle, (D) 500-grain weight, (E) yield per plant and (F) seed setting rate. 10 plants were used to measure each trait. Student's $t$-test was used for statistical analysis ( ${ }^{*} P<0.05,{ }^{* *} P<0.01$ ). Means and standard deviations are marked as values and vertical bars, respectively. (G) Fertile and sterile seeds from the whole plants of ZH11 and ZH11(e/1). Scale bar $=2 \mathrm{~cm}$. (DOCX $1723 \mathrm{~kb})$

Additional file 7: Table S1. Primers for RT-qPCR. (DOCX $22 \mathrm{~kb}$ )

\section{Abbreviations}

CKI: Casein kinase I; CYP703A3: Cytochrome P450 hydroxylase; EL1: Early flowering1; HNIL: Heterogeneous inbred family-near isogenic line; KAR: $\beta$-ketoacyl reductase; M23: Milyang23; OsPRR37: Oryza sativa pseudo-response regulator37; SLR1: Slender rice1.

\section{Competing Interests}

The authors declare that they have no competing interests.

\section{Authors' Contributions}

CTK and NCP designed the project and CTK performed all the experiments and wrote the manuscript. SHK assisted in conducting experiments, especially evaluating the data for agronomic traits. DK assisted with experiments, especially the investigation of rice floral organ structure. NCP provided the direction for the study and edited the manuscript. All authors read and approved the final manuscript.

\section{Acknowledgments}

We thank Dr. Hong-Wei Xue for donating the T-DNA insertion el1 mutant [ZH11(e/1)] seeds. This work was supported by the National Research Foundation of Korea (NRF) grant funded by the Ministry of Science, ICT and Future Planning (NRF-2011-0017308).

Received: 18 May 2015 Accepted: 13 July 2015

Published online: 24 July 2015

\section{References}

Achard P, Herr A, Baulcombe DC, Harberd NP (2004) Modulation of floral development by a gibberellin-regulated microRNA. Development 131(14):3357-3365

Aya K, Ueguchi-Tanaka M, Kondo M, Hamada K, Yano K, Nishimura M, Matsuoka M (2009) Gibberellin modulates anther development in rice via the transcriptional regulation of GAMYB. Plant Cell 21(5):1453-1472

Aya K, Suzuki G, Suwabe K, Hobo T, Takahashi H, Shiono K, Yano K, Tsutsumi N, Nakazono M, Nagamura Y, Matsuoka M, Watanabe M (2011) Comprehensive network analysis of anther-expressed genes in rice by the combination of 33 laser microdissection and 143 spatiotemporal microarrays. PLoS One 6(10):e26162

Cecchetti V, Altamura MM, Falasca G, Costantino P, Cardarelli M (2008) Auxin regulates Arabidopsis anther dehiscence, pollen maturation, and filament elongation. Plant Cell 20(7):1760-1774

Chen CB, Xu YY, Ma H, Chong K (2005) Cell biological characterization of male meiosis and pollen development in rice. J Integr Plant Biol 47(6):734-744

Cheng H, Qin LJ, Lee SC, Fu XD, Richards DE, Cao DN, Luo D, Harberd NP, Peng JR (2004) Gibberellin regulates Arabidopsis floral development via suppression of DELLA protein function. Development 131(5):1055-1064

Chhun T, Aya K, Asano K, Yamamoto E, Morinaka Y, Watanabe M, Kitano H, Ashikari M, Matsuoka M, Ueguchi-Tanaka M (2007) Gibberellin regulates pollen viability and pollen tube growth in rice. Plant Cell 19(12):3876-3888

Dai C, Xue HW (2010) Rice early flowering 1, a CKI, phosphorylates DELLA protein SLR1 to negatively regulate gibberellin signalling. EMBO J 29(11):1916-1927

Dill A, Sun TP (2001) Synergistic derepression of gibberellin signaling by removing RGA and GAl function in Arabidopsis thaliana. Genetics 159(2):777-785

Evans MM, Poethig RS (1995) Gibberellins promote vegetative phase change and reproductive maturity in maize. Plant Physiol 108(2):475-487

Fleet CM, Sun TP (2005) A DELLAcate balance: the role of gibberellin in plant morphogenesis. Curr Opin Plant Biol 8(1):77-85

Fuentes S, Ljung K, Sorefan K, Alvey E, Harberd NP, Ostergaard L (2012) Fruit growth in Arabidopsis occurs via DELLA-dependent and DELLA-independent gibberellin responses. Plant Cell 24(10):3982-3996

Gomi K, Sasaki A, Itoh H, Ueguchi-Tanaka M, Ashikari M, Kitano H, Matsuoka M (2004) GID2, an F-box subunit of the SCF E3 complex, specifically interacts with phosphorylated SLR1 protein and regulates the gibberellin-dependent degradation of SLR1 in rice. Plant J 37(4):626-634

Goto N, Pharis RP (1999) Role of gibberellins in the development of floral organs of the gibberellin-deficient mutant, ga1-1, of Arabidopsis thaliana. Can J Bot 77(7):944-954

Gubler F, Kalla R, Roberts JK, Jacobsen JV (1995) Gibberellin-regulated expression of a myb gene in barley aleurone cells: Evidence for Myb transactivation of a high-pl a-amylase gene promoter. Plant Cell 7(11):1879-1891 
Gubler F, Raventos N, Keys M, Watts R, Mundy J, Jacobsen JV (1999) Target genes and regulatory domains of the GAMYB transcriptional activator in cereal aleurone. Plant J 17(1):1-9

Gubler F, Chandler PM, White RG, Llewellyn DJ, Jacobsen JV (2002) Gibberellin signaling in barley aleurone cells. Control of SLN1 and GAMYB expression. Plant Physiol 129(1):191-200

Guo JX, Liu YG (2012) Molecular control of male reproductive development and pollen fertility in rice. J Integr Plant Biol 54(12):967-978

Hedden P, Phillips AL (2000) Gibberellin metabolism: new insights revealed by the genes. Trends Plant Sci 5(12):523-530

Hirano K, Aya K, Hobo T, Sakakibara H, Kojima M, Shim RA, Hasegawa Y, Ueguchi-Tanaka M, Matsuoka M (2008) Comprehensive transcriptome analysis of phytohormone biosynthesis and signaling genes in microspore/ pollen and tapetum of rice. Plant Cell Physiol 49(10):1429-1450

Hori K, Ogiso-Tanaka E, Matsubara K, Yamanouchi U, Ebana K, Yano M (2013) $\mathrm{Hd16}$, a gene for casein kinase $\mathrm{I}$, is involved in the control of rice flowering time by modulating the day-length response. Plant J 76(1):36-46

Hu JH, Mitchum MG, Barnaby N, Ayele BT, Ogawa M, Nam E, Lai WC, Hanada A, Alonso JM, Ecker JR, Swain SM, Yamaguchi S, Kamiya Y, Sun TP (2008) Potential sites of bioactive gibberellin production during reproductive growth in Arabidopsis. Plant Cell 20(2):320-336

Ikeda A, Ueguchi-Tanaka M, Sonoda Y, Kitano H, Koshioka M, Futsuhara Y, Matsuoka M, Yamaguchi J (2001) Slender rice, a constitutive gibberellin response mutant, is caused by a null mutation of the SLR1 gene, an ortholog of the height-regulating gene GAI/RGA/RHT/D8. Plant Cell 13(5):999-1010

Itoh H, Ueguchi-Tanaka M, Sato Y, Ashikari M, Matsuoka M (2002) The gibberellin signaling pathway is regulated by the appearance and disappearance of SLENDER RICE1 in nuclei. Plant Cell 14(1):57-70

Itoh H, Sasaki A, Ueguchi-Tanaka M, Ishiyama K, Kobayashi M, Hasegawa Y, Minami E, Ashikari M, Matsuoka M (2005a) Dissection of the phosphorylation of rice DELLA protein, SLENDER RICE1. Plant Cell Physiol 46(8):1392-1399

Itoh J, Nonomura K, Ikeda K, Yamaki S, Inukai Y, Yamagishi H, Kitano H, Nagato Y (2005b) Rice plant development: from zygote to spikelet. Plant Cell Physiol 46(1):23-47

Izhaki A, Borochov A, Zamski E, Weiss D (2002) Gibberellin regulates post-microsporogenesis processes in petunia anthers. Physiol Plant 115(3):442-447

Jacobsen SE, Olszewski NE (1991) Characterization of the arrest in anther development associated with gibberellin deficiency of the gib-1 mutant of tomato. Plant Physiol 97(1):409-414

Kaneko M, Inukai Y, Ueguchi-Tanaka M, Itoh H, Izawa T, Kobayashi Y, Hattori T, Miyao A, Hirochika H, Ashikari M, Matsuoka M (2004) Loss-of-function mutations of the rice GAMYB gene impair a-amylase expression in aleurone and flower development. Plant Cell 16(1):33-44

Koo BH, Yoo SC, Park JW, Kwon CT, Lee BD, An G, Zhang ZY, Li JJ, Li ZC, Paek NC (2013) Natural variation in OsPRR37 regulates heading date and contributes to rice cultivation at a wide range of latitudes. Mol Plant 6(6):1877-1888

Kwon CT, Yoo SC, Koo BH, Cho SH, Park JW, Zhang ZY, Li JJ, Li ZC, Paek NC (2014) Natural variation in Early flowering 1 contributes to early flowering in japonica rice under long days. Plant Cell Environ 37(1):101-112

Kwon CT, Koo BH, Kim D, Yoo SC, Paek NC (2015) Casein kinases I and 2a phosphorylate Oryza sativa pseudo-response regulator 37 (OsPRR37) in photoperiodic flowering in rice. Mol Cells 38(1):81-88

Lanahan MB, Ho THD (1988) Slender barley - a constitutive gibberellin-response mutant. Planta 175(1):107-114

Liu W, Zhang DC, Tang MF, Li DY, Zhu YX, Zhu LH, Chen CY (2013) THIS1 is a putative lipase that regulates tillering, plant height, and spikelet fertility in rice. J Exp Bot 64(14):4389-4402

Ma H (2005) Molecular genetic analyses of microsporogenesis and microgametogenesis in flowering plants. Annu Rev Plant Biol 56:393-434

Matsubara K, Kono I, Hori K, Nonoue Y, Ono N, Shomura A, Mizubayashi T, Yamamoto S, Yamanouchi U, Shirasawa K, Nishio T, Yano M (2008) Novel QTLs for photoperiodic flowering revealed by using reciprocal backcross inbred lines from crosses between japonica rice cultivars. Theor Appl Genet 117:935-945

McCormick S (2004) Control of male gametophyte development. Plant Cell 16:S142-S153

Millar AA, Gubler F (2005) The Arabidopsis GAMYB-like genes, MYB33 and MYB65, are microRNA-regulated genes that redundantly facilitate anther development. Plant Cell 17(3):705-721
Murray F, Kalla R, Jacobsen J, Gubler F (2003) A role for HvGAMYB in anther development. Plant J 33(3):481-491

Nester JE, Zeevaart JAD (1988) Flower development in normal tomato and a gibberellin-deficient (ga-2) mutant. Am J Bot 75(1):45-55

Park JH, Halitschke R, Kim HB, Baldwin IT, Feldmann KA, Feyereisen R (2002) A knock-out mutation in allene oxide synthase results in male sterility and defective wound signal transduction in Arabidopsis due to a block in jasmonic acid biosynthesis. Plant J 31(1):1-12

Plackett ARG, Thomas SG, Wilson ZA, Hedden P (2011) Gibberellin control of stamen development: a fertile field. Trends Plant Sci 16(10):568-578

Plackett ARG, Ferguson AC, Powers SJ, Wanchoo-Kohli A, Phillips AL, Wilson ZA, Hedden P, Thomas SG (2014) DELLA activity is required for successful pollen development in the Columbia ecotype of Arabidopsis. New Phytol 201(3):825-836

Ritchie S, Gilroy S (1998) Gibberellins: regulating genes and germination. New Phytol 140(3):363-383

Sasaki A, Itoh H, Gomi K, Ueguchi-Tanaka M, Ishiyama K, Kobayashi M, Jeong DH, An G, Kitano H, Ashikari M, Matsuoka M (2003) Accumulation of phosphorylated repressor for gibberellin signaling in an F-box mutant. Science 299(5614):1896-1898

Scott RJ, Spielman M, Dickinson HG (2004) Stamen structure and function. Plant Cell 16:S46-S60

Shimada A, Ueguchi-Tanaka M, Sakamoto T, Fujioka S, Takatsuto S, Yoshida S, Sazuka T, Ashikari M, Matsuoka M (2006) The rice SPINDLY gene functions as a negative regulator of gibberellin signaling by controlling the suppressive function of the DELLA protein, SLR1, and modulating brassinosteroid synthesis. Plant J 48(3):390-402

Singh DP, Jermakow AM, Swain SM (2002) Gibberellins are required for seed development and pollen tube growth in Arabidopsis. Plant Cell 14(12):3133-3147

Thangasamy S, Guo CL, Chuang MH, Lai MH, Chen JC, Jauh GY (2011) Rice SIZ1, a SUMO E3 ligase, controls spikelet fertility through regulation of anther dehiscence. New Phytol 189(3):869-882

Tsuji H, Aya K, Ueguchi-Tanaka M, Shimada Y, Nakazono M, Watanabe R, Nishizawa NK, Gomi K, Shimada A, Kitano H, Ashikari M, Matsuoka M (2006) GAMYB controls different sets of genes and is differentially regulated by microRNA in aleurone cells and anthers. Plant J 47(3):427-444

Ueguchi-Tanaka M, Ashikari M, Nakajima M, Itoh H, Katoh E, Kobayashi M, Chow TY, Hsing YIC, Kitano H, Yamaguchi I, Matsuoka M (2005) GIBBERELLIN INSENSITIVE DWARF1 encodes a soluble receptor for gibberellin. Nature 437(7059):693-698

Ueguchi-Tanaka M, Nakajima M, Katoh E, Ohmiya H, Asano K, Saji S, Xiang HY, Ashikari M, Kitano H, Yamaguchi I, Matsuokaa M (2007a) Molecular interactions of a soluble gibberellin receptor, GID1, with a rice DELLA protein, SLR1, and gibberellin. Plant Cell 19(7):2140-2155

Ueguchi-Tanaka M, Nakajima M, Motoyuki A, Matsuoka M (2007b) Gibberellin receptor and its role in gibberellin signaling in plants. Annu Rev Plant Biol 58:183-198

Wilson ZA, Zhang DB (2009) From Arabidopsis to rice: pathways in pollen development. J Exp Bot 60(5):1479-1492

Woodger FJ, Millar A, Murray F, Jacobsen JV, Gubler F (2003) The role of GAMYB transcription factors in GA-regulated gene expression. J Plant Growth Regul 22(2):176-184

Yamaguchi J (1998) Analysis of embryo-specific alpha-amylase using isolated mature rice embryos. Breed Sci 48(4):365-370

Zhu QH, Ramm K, Shivakkumar R, Dennis ES, Upadhyaya NM (2004) The ANTHER INDEHISCENCE1 gene encoding a single MYB domain protein is involved in anther development in rice. Plant Physiol 135(3):1514-1525 